\title{
Feasibility of Emergency Laparoscopic Reoperations for Complications after Laparoscopic Surgery for Colorectal Cancer
}

\author{
Chang Woo Kim, M.D., Suk-Hwan Lee, M.D., Ph.D. \\ Department of Surgery, Kyung Hee University Hospital at Gangdong, Kyung Hee University School of Medicine, Seoul, Korea
}

Purpose: Laparoscopic surgery (LS) is an alternative to colorectal cancer surgery. Little evidence supports LS for emergency reoperation after laparoscopic colorectal surgery. The aim of this study was to assess perioperative outcomes of LS as an emergency reoperation for early complications after LS for colorectal cancer.

Methods: From June 2006 through December 2016, 732 consecutive patients underwent elective LS for colorectal cancer at Kyung Hee University Hospital, Seoul, Korea. Among these patients, we retrospectively reviewed data on those who received emergency laparoscopic reoperations for complications within 30 days after surgery. Variables associated with perioperative outcomes were analyzed.

Results: After exclusion of 50 patients (6.8\%) who needed conversion to open surgery during LS, 79 of 682 patients (11.6\%) received reoperation for complications, recurrence, and other benign diseases. Among them, 22 patients underwent emergency laparoscopic reoperation for early complications. Mean age of the patients was 62 years, and most underwent low anterior resection as a primary operation $(n=17,77.3 \%)$. Anastomotic leakage was the most common reason for reoperation $(n=14$, 63.6\%). Postoperative complication occurred in 6 patients (27.3\%), but none required further surgical intervention. Patients had first bowel movements at 2.8 days after reoperation, and length of hospital stay was 17.2 days after reoperation.

Conclusion: Laparoscopic reoperation showed acceptable outcomes. LS as a reoperation for complications seemed to be feasible after LS for colorectal cancer.

Keywords: Laparoscopic surgery, Minimally invasive surgery, Colon cancer, Rectal cancer, Reoperation
Received November 9, 2017

Revised 1st December 15, 2017 2nd January 31, 2018

Accepted February 1, 2018

Corresponding author

Suk-Hwan Lee

Department of Surgery, Kyung Hee University Hospital at Gangdong, Kyung Hee University School of Medicine, 892 Dongnam-ro, Gangdong-gu, Seoul 05278, Korea Tel: +82-2-440-6134

Fax: +82-2-440-6073

E-mail: leeshdr@gmail.com ORCID:

http://orcid.org/0000-0001-6470-8620

This is an Open Access article distributed under the terms of the Creative Commons Attribution Non-Commercial License (http:// creativecommons.org/licenses/by-nc/4.0/) which permits unrestricted non-commercial use, distribution, and reproduction in any medium, provided the original work is properly cited.

Copyright @ 2018 The Journal of Minimally Invasive Surgery. All rights reserved.

\section{INTRODUCTION}

Minimally invasive surgery (MIS) is used for colorectal cancer based on comparable short-term and long-term outcomes. Laparoscopic surgery (LS) has advantages over open surgery (OS) including smaller incision, less blood loss, and shorter length of stay. ${ }^{1-4}$ Two papers analyzing data from the National Surgical Quality Improvement Program reported that LS decreases postoperative complication rates and reduces surgical site infections. ${ }^{5,6}$ However, a significant proportion of patients need reoperations due to postoperative complications, recurrences, intestinal obstruction, or other intraabdominal diseases. When reoperation is needed after colorectal cancer surgery, OS is preferred because of obstacles to reoperation such as small bowel distention, postoperative adhesion, or lack of adequate operative field. However, accumulation of experience in LS changes the concept of reoperation. Even though intraabdominal conditions are not ideal for performing LS 
in emergency conditions, once LS is conducted, postoperative recovery would be better than OS. LS could be an option for reoperation as well as for primary surgery. Many surgeons perform LS for reoperations after LS and after OS based on their clinical experience. These surgeons would like to offer the benefits of MIS in emergency situations. Several papers have reported comparable outcomes for LS as a reoperation in various situations. ${ }^{5,8-11}$ The aim of this study was to assess perioperative outcomes of LS as an emergency reoperation for complications within 30 days after LS for colorectal cancer.

\section{MATERIALS AND METHODS}

\section{Patients}

From June 2006 through December 2016, 732 consecutive patients underwent elective LS for colorectal cancer at Kyung Hee University Hospital, Seoul, Korea. Among these patients, those who received elective reoperations for recurrence $(n=38)$, stoma takedown $(n=2)$, or other benign diseases $(n=9)$ were excluded. Patients who received OS as a reoperation or any reoperation for late complications ( $>30$ days after primary surgery, $\mathrm{n}=6$ ) were also excluded. Data on patients who received emergency laparoscopic reoperation for complications within 30 days after primary LS were reviewed retrospectively.

\section{Perioperative outcomes}

Data on gender, age, body mass index, American Society of Anesthesiologists grade, previous abdominal surgery, and location of primary lesion were analyzed for baseline characteristics of patients. For analysis of perioperative outcomes of primary surgery, operation time (OT), intraoperative complication, and length of hospital stay (LOS) were analyzed. For analysis of reoperation outcomes, variables analyzed in addition to the variables for primary surgery were postoperative complication after primary surgery (cause of reoperation), hemodynamic stability at time of reoperation, OT, blood loss, intraoperative complication, time to first bowel movement, time to resumption of soft diet, and postoperative LOS. Hemodynamic instability was defined as body temperature $>38^{\circ} \mathrm{C}$, mean blood pressure $<70 \mathrm{~mm} \mathrm{Hg}$, or heart rate $>100$ per minute.

\section{RESULTS}

\section{Primary surgery}

This study analyzed 22 patients who underwent LS due to complications within 30 days of primary surgery $(22 / 732=3 \%)$.
Baseline characteristics of patients and data on primary surgery are shown in Table 1: $81.8 \%$ of patients were men, and the mean age was 62 years. The main primary tumor location was the rectum (63.6\%), and the mean OT was 279.2 minutes. The most common postoperative complication that required reoperation was anastomotic leakage $(n=14,63.6 \%)$. The duration between primary surgery and reoperation was 8.6 days. Mean length of hospital stay after primary surgery was 25.8 days. For patients discharged without complications after LS, postoperative length of stay was $8.1 \pm 2.1$ days (data not shown).

Table 1. Baseline characteristics and perioperative outcomes Iprimary surgeryl

\begin{tabular}{lc}
\hline \multicolumn{1}{|c}{ Variable } & $\mathrm{n}=22$ \\
\hline Male gender & $18(81.8 \%)$ \\
\hline Age lyears) & $62 \pm 10.4$ \\
\hline BMI (kg/m $\left.{ }^{2}\right)$ & $23.4 \pm 3.6$ \\
\hline ASA grade & \\
\hline I & $8(36.4 \%)$ \\
\hline II & $11(50 \%)$ \\
\hline III & $3(13.6 \%)$ \\
\hline Previous abdominal surgery & $5(22.7 \%)$ \\
\hline Location of primary lesion & \\
\hline Ascending colon & $1(4.5 \%)$ \\
\hline Transverse colon & $1(4.5 \%)$ \\
\hline Descending colon & $1(4.5 \%)$ \\
\hline Sigmoid colon & $4(18.1 \%)$ \\
\hline Rectum & $14(63.6 \%)$ \\
\hline Synchronous & $1(4.5 \%)$ \\
\hline Curative resection (RO) & $21(95.5 \%)$ \\
\hline Combined resection & $3(13.6 \%)$ \\
\hline Operation time of primary surgery (minutes) & $279.2 \pm 148.5$ \\
\hline Postoperative complications & \\
\hline Anastomotic leakage & $14(63.6 \%)$ \\
\hline Bleeding & $3(13.6 \%)$ \\
\hline Colon ischemia & $2(9.1 \%)$ \\
\hline Small bowel obstruction & $1(4.5 \%)$ \\
\hline Small bowel perforation & $1(4.5 \%)$ \\
\hline Stoma necrosis & $1(4.5 \%)$ \\
\hline Postoperative length of stay (days) & $25.8 \pm 11.7$ \\
\hline B bod & \\
\hline
\end{tabular}

$\mathrm{BMI}=$ body mass index; $\mathrm{ASA}=$ American Society of Anesthesiologists. 


\section{Reoperation}

Demographics of patients who underwent laparoscopic reoperation are presented in Table 2. Hemodynamic instability was noted in 17 patients (77.3\%). Reoperation types were abdominoperineal resection $(n=2)$, bleeding control $(n=3)$, Hartmann operation $(n=1)$, primary repair of anastomotic dehiscence $(n=2)$, stoma formation with or without peritoneal irrigation $(n=13)$, and stoma revision $(n=1)$. Mean operation time was 125 minutes with one conversion to OS for severe dilatation of the small bowel. Small bowel injuries were found during surgery in two patients and were repaired by laparoscopy intracorporeally. We noted six postoperative complications $(27.3 \%)$ of ileus, wound infection, or hematochezia; none required surgical intervention. Patients showed first bowel movement at postoperative day (POD) 2.8 and started a soft diet at POD 6.8. Mean length of hospital stay after reoperation was 17.2 days which was shorter than in patients who underwent $\mathrm{OS}$ as a reoperation ( $22.2 \pm 7.6$ days). There was no mortality after reoperation.

Table 2. Perioperative outcomes (reoperation)

\begin{tabular}{lc}
\hline \multicolumn{1}{c}{ Variable } & $\mathrm{n}=22$ \\
\hline Reoperation & \\
Abdominoperineal resection & $2(9.1 \%)$ \\
Bleeding control & $3(13.6 \%)$ \\
Hartmann operation & $1(4.5 \%)$ \\
Primary repair & $2(9.1 \%)$ \\
Stoma formation with or without peritoneal irrigation & $13(59.1 \%)$ \\
Stoma revision & $1(4.5 \%)$ \\
\hline Date from the primary surgery (days) & $5.5(1-30)^{*}$ \\
Unstable hemodynamics & $17(77.3 \%)$ \\
Conversion & $1(4.5 \%)$ \\
Operation time (minutes) & $125 \pm 79.9$ \\
Blood loss (ml) & $10(0-1180)^{*}$ \\
Postoperative complication & $6(27.3 \%)$ \\
Small bowel ileus & $2(9.1 \%)$ \\
Wound infection & $3(13.6 \%)$ \\
Hematochezia & $1(4.5 \%)$ \\
ICU stay (days) & $0.9 \pm 1.4$ \\
Pirst flatus (days) & $2.8 \pm 1.5$ \\
\hline & $6.8 \pm 4.7$ \\
\hline & $17.2 \pm 8.8$ \\
\hline
\end{tabular}

*Median (range). ICU = intensive care unit.

\section{DISCUSSION}

LS is regarded as a safe and feasible option for primary surgery for colorectal cancer. Laparoscopic emergency reoperation has obstacles such as bowel edema, postoperative ileus, and poor operative field. Many surgeons prefer OS to LS in emergency conditions for several reasons. First, primary abdominal surgery can result in adhesion that might interfere with LS and result in OS or conversion to OS. Second, conversion to OS during LS might require a longer operation time, longer hospital stay, and higher cost. Third, performing LS in emergency situations such as peritonitis, ischemia or bowel perforation can increase the risk of hypercapnia and toxic shock syndrome. ${ }^{12}$ Therefore, the advantages of LS should be considered both for reoperation as well as primary surgery.

Stoma formation with or without peritoneal irrigation (59.1\%) and bleeding control (13.6\%) were performed as reoperations in our study. Most reoperations were less difficult procedures than the primary surgery except for two abdominoperineal resections (9.1\%). Reoperations were performed to control complications and did not require long OTs. Moreover, LS as reoperation did not yield additional surgical incisions except for trocar sites after primary surgery in this study. Using the same incision sites should be emphasized as pros of relaparoscopy that might result in early recovery and less wound complications. Therefore, we suggest that LS should be considered as a reoperation method.

Conversion rates were similar for primary surgery and reoperation $(6.8 \%=50$ of 720 primary and $4.5 \%=1$ of 22 reoperations) in our study. In the Clinical Outcomes of Surgical Therapy Study Group, conversion rate to OS during LS was $21 \%$. Among reasons for conversion, adhesion represented about $3 \%$ of cases. ${ }^{2}$ In the MRC CLASICC trial that analyzed a conversion group and OS and LS groups, the conversion group (29\%) showed longer extended hospital stays than the LS group. The most common causes of conversion were excessive tumor fixity and uncertainty of tumor clearance, not adhesion. ${ }^{3}$ Adhesion alone was not the reason for conversion. Burns et al. reported that patients who underwent LS for colorectal diseases had a lower risk of developing clinically significant adhesions compared with OS. ${ }^{11}$ These results suggest that adhesion is not an absolute contraindication for LS, and there are techniques to treat adhesion laparoscopically. Conversion to open surgery during reoperation in our study occurred in $4.8 \%$ of cases $(n=1)$ and was due to bowel distention.

Hemodynamic instability of the patient can make surgeons hesitate select laparoscopy as reoperation method. In this study, $77.3 \%$ of the patients showed hemodynamic instability. However, most of them showed fever or tachycardia, whereas 
no patient showed shock status (mean blood pressure $<70$ $\mathrm{mmHg}$ ). This is one of the limitations of the study. In spite of several concerns, LS in emergency situations appears feasible. Navez et al. concluded that LS is feasible based on acceptable morbidity and mortality rates without malignant hypercapnia. ${ }^{12}$ Agresta and colleagues reported that LS is not an alternative to physical examination or noninvasive diagnostic methods but must be considered an effective option as an alternative to OS. ${ }^{13}$ Our study involved six postoperative complications, none of which required surgical intervention.

Several studies have reported outcomes of LS as reoperation. Rosin et al. showed acceptable outcomes for 14 patients who received LS as reoperation for complications after OS. ${ }^{14}$ Rotholtz and colleagues reported that LS is a useful tool for managing complications after LS for colorectal disease.10 Kirshtein et al. compared outcomes of patients according to timing of relaparoscopy and concluded that early relaparoscopy enabled timely management of complications. ${ }^{9}$ In a study limited to anastomotic leakage for relaparoscopy, Vennix et al. evaluated safety and feasibility of LS as a reoperation after colorectal surgery based on shorter hospital stay, fewer complications, and less mortality compared with OS. ${ }^{15}$ Lee et al. also reported that relaparoscopy is associated with shorter hospital stay and fewer complications than OS for anastomotic leakage. ${ }^{16}$ Anastomotic leakage is one of the most fatal complications after rectal surgery rather than colon surgery, and most of them requires surgical intervention. Laparoscopy might be an option for anastomotic leakage. However, most studies that reported outcomes of relaparoscopy had a small number of cases or patients with heterogeneous disease characteristics. These problems are also limitations of our study, since reoperation itself should be infrequent in order to ensure patient safety.

Our study has several limitations. First, it was a retrospective study with the possibility of selection bias. Second, there were a small number of patients and heterogeneous reasons for reoperation. Third, if we compared LS and OS as reoperation methods, the safety and feasibility of LS would be clearer. However, this comparison would not provide meaningful results because of the small number of OS cases $(n=3)$ in our clinical practice.

In conclusion, LS as reoperation method seemed feasible because it did not require many open conversions and did not result in poor short-term outcomes. LS might provide the advantages of MIS even after reoperation. Surgeons should consider LS as a reoperation method.

\section{ACKNOWLEDGMENTS}

This work was supported by the National Research Founda- tion of Korea (NRF) grant funded by the Korea Government Ministry of Education (No. 017R1D1A1B03030948).

\section{REFERENCES}

1) Lacy AM, Garcia-Valdecasas JC, Delgado S, et al. Laparoscopyassisted colectomy versus open colectomy for treatment of nonmetastatic colon cancer: a randomised trial. Lancet 2002;359:22242229.

2) Clinical Outcomes of Surgical Therapy Study Group, Nelson H, Sargent DJ, et al. A comparison of laparoscopically assisted and open colectomy for colon cancer. N Engl J Med 2004;350:20502059.

3) Guillou PJ, Quirke P, Thorpe H, et al. Short-term endpoints of conventional versus laparoscopic-assisted surgery in patients with colorectal cancer (MRC CLASICC trial): multicentre, randomised controlled trial. Lancet 2005;365:1718-1726.

4) Jayne DG, Thorpe HC, Copeland J, Quirke P, Brown JM, Guillou PJ. Five-year follow-up of the Medical Research Council CLASICC trial of laparoscopically assisted versus open surgery for colorectal cancer. Br J Surg 2010;97:1638-1645.

5) Kennedy GD, Heise C, Rajamanickam V, Harms B, Foley EF. Laparoscopy decreases postoperative complication rates after abdominal colectomy: results from the national surgical quality improvement program. Ann Surg 2009;249:596-601.

6) Kiran RP, El-Gazzaz GH, Vogel JD, Remzi FH. Laparoscopic approach significantly reduces surgical site infections after colorectal surgery: data from national surgical quality improvement program. J Am Coll Surg 2010;211:232-238.

7) Wang L, Yan H, Li J. Outcomes of laparoscopic colorectal surgery in patients of previous abdominal surgery: a systemic review and meta-analysis. Hepatogastroenterology 2015;62:273-278.

8) Wind J, Koopman AG, van Berge Henegouwen MI, Slors JF, Gouma DJ, Bemelman WA. Laparoscopic reintervention for anastomotic leakage after primary laparoscopic colorectal surgery. Br J Surg 2007;94:1562-1566.

9) Kirshtein B, Roy-Shapira A, Domchik S, Mizrahi S, Lantsberg L. Early relaparoscopy for management of suspected postoperative complications. J Gastrointest Surg 2008;12:1257-1262.

10) Rotholtz NA, Laporte M, Lencinas SM, Bun ME, Aued ML, Mezzadri NA. Is a laparoscopic approach useful for treating complications after primary laparoscopic colorectal surgery? Dis Colon Rectum 2009;52:275-279.

11) Burns EM, Currie A, Bottle A, Aylin P, Darzi A, Faiz O. Minimalaccess colorectal surgery is associated with fewer adhesion-related admissions than open surgery. Br J Surg 2013;100:152-159.

12) Navez B, Tassetti V, Scohy JJ, et al. Laparoscopic management of acute peritonitis. Br J Surg 1998;85:32-36.

13) Agresta F, Mazzarolo G, Ciardo LF, Bedin N. The laparoscopic approach in abdominal emergencies: has the attitude changed?: A 
single-center review of a 15-year experience. Surg Endosc 2008; 22:1255-1262.

14) Rosin D, Zmora O, Khaikin M, Bar Zakai B, Ayalon A, Shabtai M. Laparoscopic management of surgical complications after a recent laparotomy. Surg Endosc 2004;18:994-996.

15) Vennix S, Abegg R, Bakker OJ, et al. Surgical re-interventions following colorectal surgery: open versus laparoscopic management of anastomotic leakage. J Laparoendosc Adv Surg Tech A 2013;23:739-744.

16) Lee CM, Huh JW, Yun SH, et al. Laparoscopic versus open reintervention for anastomotic leakage following minimally invasive colorectal surgery. Surg Endosc 2015;29:931-936. 